The Astrophysical Journal, 609:1140-1152, 2004 July 10

(C) 2004. The American Astronomical Society. All rights reserved. Printed in U.S.A.

\title{
SOLAR CYCLE 23: AN ANOMALOUS CYCLE?
}

\author{
Giuliana de Toma and Oran R. White \\ High Altitude Observatory, National Center for Atmospheric Research, Boulder, CO 80301; detoma@ucar.edu, orw@hao.ucar.edu
}

AND

Gary A. Chapman, Stephen R. Walton, Dora G. Preminger, and Angela M. Cookson

San Fernando Observatory, Department of Physics and Astronomy, California State University at Northridge, 18111 Nordhoff Street, Northridge, CA 91330; gchapman@csun.edu, stephen.walton@csun.edu, dora.preminger@csun.edu, angie.cookson@csun.edu

Received 2004 January 24; accepted 2004 March 19

\begin{abstract}
The latest $\mathrm{SOHO}$ VIRGO total solar irradiance (TSI) time series is analyzed using new solar variability measures obtained from full-disk solar images made at the San Fernando Observatory and the Mg II $280 \mathrm{~nm}$ index. We discuss the importance of solar cycle 23 as a magnetically simpler cycle and a variant from recent cycles. Our results show the continuing improvement in TSI measurements and surrogates containing information necessary to account for irradiance variability. Use of the best surrogate for irradiance variability due to photospheric features (sunspots and faculae) and chromospheric features (plages and bright network) allows fitting the TSI record to within an rms difference of $130 \mathrm{ppm}$ for the period 1986 to the present. Observations show that the strength of the TSI cycle did not change significantly despite the decrease in sunspot activity in cycle 23 relative to cycle 22 . This points to the difficulty of modeling TSI back to times when only sunspot observations were available.
\end{abstract}

Subject headings: Sun: activity — Sun: faculae, plages — sunspots

\section{INTRODUCTION}

As the result of space experiments that began in 1978, we now have a continuous record of the total solar irradiance (TSI) beginning at the maximum of solar cycle 21 , covering all of cycle 22 , and continuing through the maximum of cycle 23 (Fröhlich 2003a, 2003b, and references therein). This composite TSI record is accompanied by daily recording of fulldisk solar images at the San Fernando Observatory (SFO) from 1985 to the present. Existence of these two parallel records gives us the opportunity to relate variability of the total solar radiative input to the Earth to changes in solar features appearing on the solar disk over all of cycle 22 and through the maximum of cycle 23 . Such studies began immediately with the first TSI measurements from the Nimbus 7/ERB and SMM/ ACRIMI experiments in 1979 and 1980 because the connection to sunspots was so clear in these early records (Willson et al. 1981). Short-term TSI variations visible as rapid and deep decreases in intensity were soon explained by changes in photospheric flux caused by the passage of dark sunspots across the solar disk, while the increase preceding and following a sunspot passage was identified with faculae most visible at the limb (Willson et al. 1981). It was also recognized that dark sunspots alone cannot account for the solar cycle profile in TSI time series; bright features in active regions are required. Soon after these effects were discovered, empirical models of TSI were developed by several groups (e.g., Foukal \& Lean 1988; Lean \& Foukal 1988; Fröhlich \& Pap 1989; Willson \& Hudson 1991). These early models used a photometric sunspot index to remove the sunspot-blocking effect and a chromospheric index to represent faculae and plages. Solar images from the SFO program have good photometric calibration that allows quantitative determination of the fractional contribution of both bright and dark solar surface features to the total irradiance (Chapman et al. 1992). Solar cycle 23 is the best observed solar cycle to date, with TSI data from $\mathrm{SOHO}$ VIRGO starting in 1996, ACRIMSat/ACRIMIII starting in 2000, and SORCE/TIM starting in 2003, and the extensive image database from SFO, the National Solar Observatory (NSO), and SOHO. Thus, we combine the TSI record with that of the appearance of the solar disk each day and thereby assess how well we recover TSI variability from these independently measured time series.

We see two goals in this research. First, since there should be reasonable consistency between the irradiance record and a surrogate based on observations of the solar disk, lack of such agreement can point to systematic errors in either record. Second, we ask whether TSI variability can be accounted for using only the variation due to magnetic features such as sunspots, faculae, and remnants in the chromospheric network.

Since our surrogate observations are all on relative intensity scales, we regress linear combinations of these indices on the TSI record. This least-squares process establishes the absolute irradiance scale for the surrogate and gives the weights for each of its components. We also divide the irradiance record into segments in cycle 22 and 23 to determine if there is any variation in the nature of the source of TSI variation from cycle to cycle. We test combinations of two types of indices: (1) indices that represent irradiance decreases due to dark sunspots and increases due to bright features such as faculae and plages, and (2) indices that are integrals over the full disk of the Sun and do not require decomposition of images into different structure types.

An important finding is the consistency between the TSI record and our regression fits due to the improvement in both TSI and the activity indices time series in the last two years. Analysis of these new observational data resolves the questions about differences in TSI measurements and empirical estimates between solar cycles 22 and 23 raised in our earlier study 
(de Toma et al. 2001, hereafter Paper I). We now find that the TSI increase from solar minimum to maximum in cycle 23 agrees with estimates from irradiance indices. We are able to fit the TSI record from 1986 to the present to rms accuracies comparable to that reported for precision of the latest TSI measurements, provided we use indices containing all information from the solar disk. Our best fits come from the new fulldisk indices developed at SFO and the $\mathrm{Mg}$ II $280 \mathrm{~nm}$ index.

We also find that cycle 23 does not behave as predicted: it is a magnetically weaker and simpler cycle than its two predecessors. Sunspot emergence decreases by $\approx 37 \%$ relative to cycle 22 with an even larger decrease in the area of bright faculae and plages. Despite this decrease in coverage of the disk by these surface features, TSI values during the maximum phase of cycle 23 are comparable to those measured in the more active cycle 22 . This is a finding critical in our understanding of the differences in lifetimes of dark and bright structures as they determine the profile of TSI change over the $11 \mathrm{yr}$ Schwabe cycle. It also indicates that reconstructions of TSI back in time, when only the sunspot record was available, are likely to have large uncertainties.

Finally, we find that a quasi-periodic TSI variation with a period very close to $1 \mathrm{yr}$ occurs between 2000 and 2003. It cannot be accounted for by errors in orbital determinations for either SOHO or ACRIMSat. This periodicity also remains with a lower amplitude in the residual between TSI and our best surrogate. Although this annual variation is then not completely accounted for in our analysis, we suggest that it may originate from the timing of sunspot emergence in the maximum of cycle 23 .

The following four sections in our paper discuss properties of the solar data, characteristics of cycle 23, our regression analysis, and these findings in more detail.

\section{CHARACTER OF SOLAR CYCLE 23}

Solar cycle 23 reached its maximum during the years 20002002 , and it is now in its declining phase. It is a cycle with modest activity in comparison with the two previous solar cycles, 21 and 22. Figure 1 shows the variation in familiar solar indices in cycles 22 and 23, i.e., magnetic flux, sunspot area, facular area, $\mathrm{Mg}$ II index, and $10.7 \mathrm{~cm}$ radio flux (F10.7), together with TSI in the period from 1985 to 2003. Cycle 23 is weaker than its preceding (even numbered) cycle in all these solar indices but not in TSI. In spite of the lower activity level during cycle 23 , the average TSI value during the maximum phase of cycle 23 is comparable to that observed in cycle 22 . Table 1 gives average values of sunspot area, facular area, $10.7 \mathrm{~cm}$ radio flux, and $\mathrm{Ca}$ II and $\mathrm{Mg}$ II indices, as well as TSI and magnetic flux during the minimum and maximum phases of cycles 22 and 23. We use the yearly averages for 1986 and 1996 for solar minimum and the periods 1989-1991 and 2000-2002 for the solar maximum phase during cycle 22 and 23, respectively. The last column in Table 1 gives the ratio of change from solar minimum to solar maximum for cycle 22 and 23 to illustrate better the higher level of solar activity during cycle 22. Photospheric magnetic flux and most indices of solar activity show lower values at the maximum of cycle 23 compared with the maximum phase of cycle 22 . Cycle 23 is particularly weak in sunspot and facular activity. We note a decrease of $37 \%$ and $46 \%$ in sunspot and facular area, respectively, for cycle 23 with respect to cycle 22 . Such a low level of sunspot activity was not expected in cycle 23 .

Predictions for cycle 23 indicated a high activity level, similar to or exceeding that in cycle 22. In 1996, a panel reviewed predictions for solar cycle 23 (Joselyn et al. 1997) on the basis of precursor techniques, the even-odd rule, and statistical analysis of preceding cycles (see Hathaway et al. 1999 for a comprehensive review of prediction techniques). The panel's consensus prediction was for solar cycle 23 to be another relatively strong cycle, with maximum values of 160 for the smoothed sunspot number and 205 for the smoothed F10.7 radio flux, to occur in the spring of 2000 (Joselyn et al. 1997). The observed maximum values were instead 120.8 for the smoothed sunspot number in 2000 April and 196.8 for the smoothed F10.7 radio flux in 2002 February. These two periods in spring 2000 and early 2002 correspond to two relative maxima shown by the activity indices in cycle 23 (see Fig. 1).

Cycle 23 may be considered anomalous because it violates the Gnevyshev \& Ohl (1948) even-odd rule found to hold for $150 \mathrm{yr}$ in the Zurich sunspot number record. This empirical rule states that an odd-numbered solar cycle is stronger in annual mean sunspot number than its preceding even-numbered cycle. The Zurich sunspot number record follows this empirical rule starting with cycle 9 until cycle 21 . However, violations of the odd-even rule may have occurred in the past as inferred from the historical record of aurorae (Schove 1955; Komitov \& Bonev 2001) for other odd-numbered cycles following particularly strong even-numbered cycles. Interestingly, this violation of the even-odd rule for cycles 22 and 23 was predicted almost 50 years ago by Schove (1955) and recently by Komitov \& Bonev (2001) using a similar database. This departure of sunspot activity in cycle 23 from its recent pattern raises questions about the physical basis for the evenodd rule. Flux transport solar dynamo models with realistic meridional circulation indicate that the strength of a cycle is affected by the two preceding cycles and do not support the even-odd rule often used to predict the strength of the next odd-numbered cycle. Instead, model simulations show that cycle $n-2$ and not cycle $n-1$ has the greater influence on a given solar cycle $n$ (Charbonneau \& Dikpati 2000). This relationship between cycles $n$ and $n-2$ has been confirmed by a recent analysis of the sunspot record (D. H. Hathaway 2003, private communication).

\subsection{Variations in Total Solar Irradiance}

In addition to the lower sunspot production in cycle 23 described above, Livingston (2002) pointed out that sunspot umbral intensities in cycle 23 are brighter and have a lower magnetic flux on average than in cycle 22 . The lack of large and dark sunspot groups in cycle 23 is clear in the TSI time series. We see several large dips in cycle 22 TSI data corresponding to the passage of large sunspot groups across the solar disk. Such deep dips are less common in the TSI data for cycle 23 (see Fig. 1). There are only six active regions in cycle 23 from 1998 to 2002 with area greater than 1400 millionths of the hemisphere compared with over 30 active regions of this size in cycle 22 between 1988 and $1992 .^{1}$

Two of the most prominent sunspot groups in cycle 23 correspond to the large active regions in 2000 September (NOAA AR 9169) and 2001 March (NOAA AR 9393). Both active regions were preceded by very low solar activity. In

\footnotetext{
1 Three additional active regions, AR 10484, AR 10486, and AR 10488, with area larger than 1400 millionths of the hemisphere appeared in 2003 October. This short period of intense solar activity, which is not included in the present study, was the largest episode of sunspot emergence to date in cycle 23 .
} 
Total Solar Irradiance (composite)

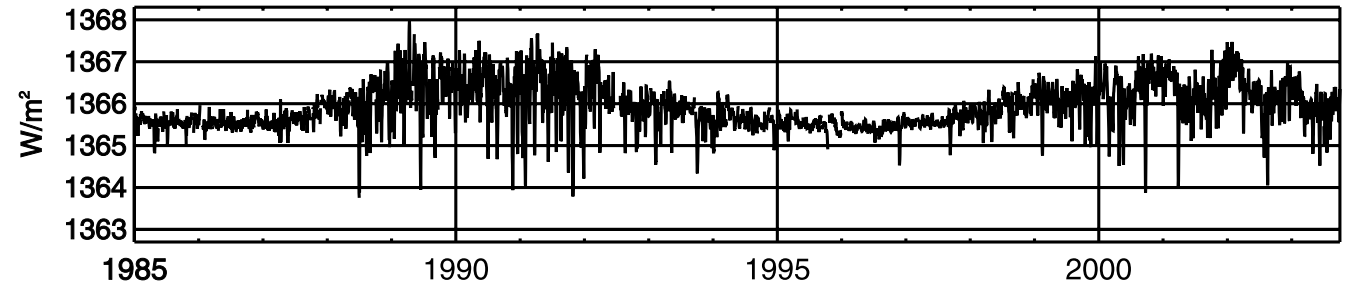

Magnetic Flux
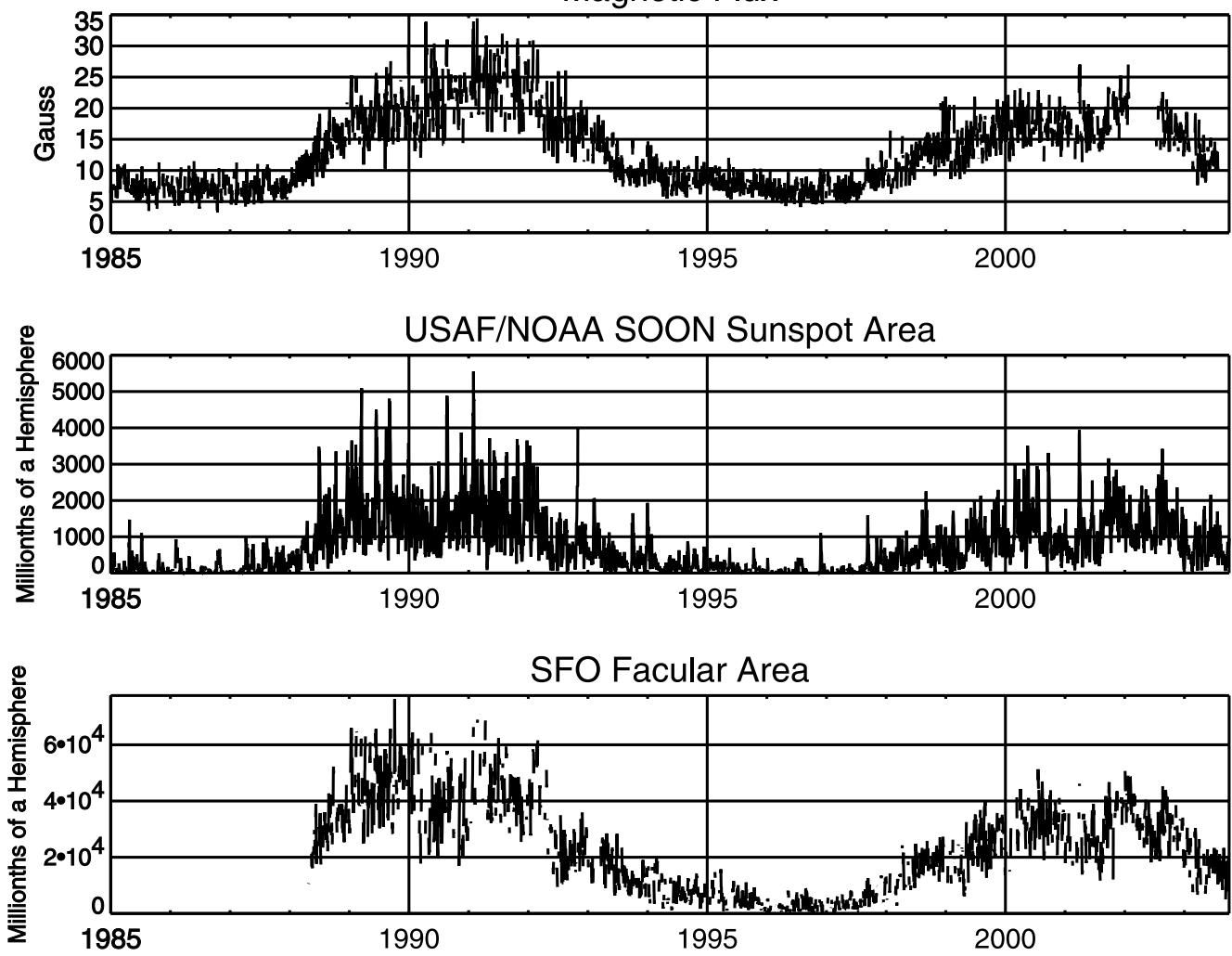

Mgll 280nm index (composite)

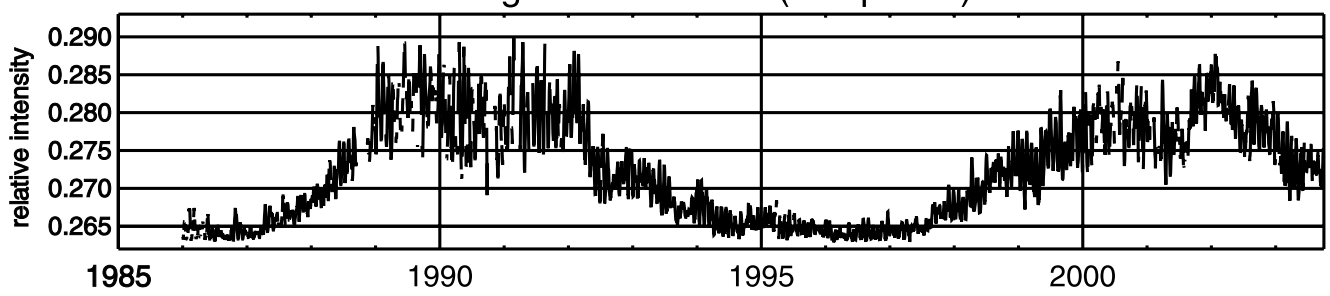

$10.7 \mathrm{~cm}$ Radio Flux

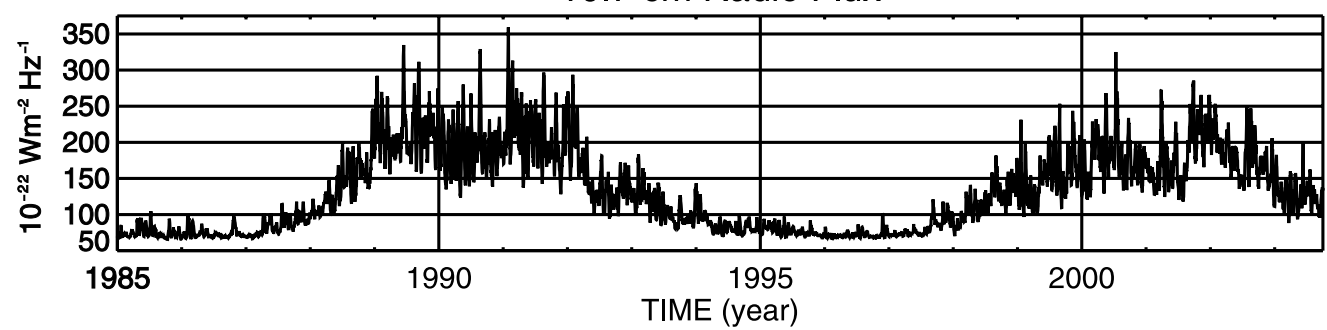

FIG. 1.-Time series of daily values for TSI (composite), magnetic flux, sunspot area, facular area, magnesium index (composite), and radio flux at 10.7 cm during the years 1985-2003. The period covers the solar cycles 22 and 23 . We note the significant decrease in sunspot and magnetic activity during cycle 23. 
TABLE 1

Solar Activity Indices and Total Solar Irradiance: Minimum to Maximum Variability

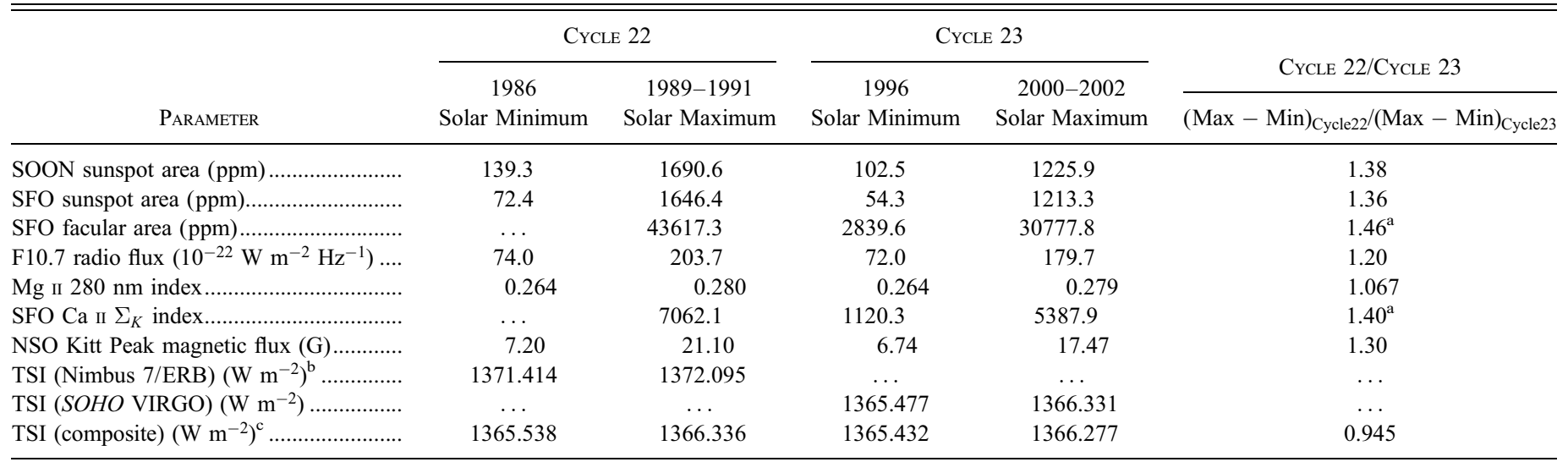

NoтEs.- The table shows the average values during the solar maximum and minimum phase for solar cycles 22 and 23 . The ratio in the last column illustrates the difference in the rate of increase from minimum to maximum between the two cycles for seven activity parameters and the TSI composite.

a SFO facular areas and the $\Sigma_{K}$ index are not available in 1986 since the SFO observing program in the Ca II K line started in 1988 . The observed minimum value in 1996 has been used to estimate the minimum in 1986.

b Nimbus7/TSI data have been provided by G. A. Chapman.

c TSI composite record compiled by C. Fröhlich and his collaborators (Fröhlich \& Lean 1998a; Fröhlich 2000, 2003b).

particular, sunspot activity was greatly reduced between 2000 October and 2001 March (see Fig. 2). Lower values for the total photospheric magnetic flux and other solar activity indices were also observed in this period. TSI values, however, remained high throughout the entire period. It is well known that magnetic flux and TSI are not well correlated on intermediate and short timescales. The relatively low values of sunspot and plage indices during this period and the high values of TSI illustrate the importance of understanding magnetic flux emergence in sunspots, faculae or plages, and network as sources of TSI variability due to competing effects of dark and bright regions on the solar disk.

Short-term TSI variability is due to a competition between dark sunspots and bright faculae or plages. At solar minimum, when sunspots are largely absent, chromospheric radiation is weak for lack of emission from plages. From 2000 October to 2001 March, we see reduced sunspot activity, but the longer lived bright faculae and plages stay at a relatively high level (see Fig. 2). The lack of new sunspots is likely the reason TSI remains high while indices directly related to sunspots fall. This indicates that TSI values are more sensitive to the balance between the radiation from sunspots, faculae, and plages than to the level of total magnetic flux alone.

In Figure 2 we plot daily values and weighted averages over a period of 81 days for TSI, sunspot disk area, and the Mg II index. This smoothed TSI record shows three peaks during the maximum phase of cycle 23 . The first peak coincides with the period between 2000 October and 2001 March described above. The second and third peaks occur in early 2002 and early 2003, about a year apart. The high value of TSI in early 2002 is the result of relatively few sunspots and greater facular area. Facular area reached a maximum in early 2002. As a result, indices of solar activity dominated by chromospheric radiation from plages, such as the $\mathrm{Mg}$ II and the $\mathrm{Ca}$ II $\mathrm{K}$ indices, also showed a maximum at this time (see Figs. 1 and 2). The third peak in TSI in early 2003 is similar to that in 20002001. Significant sunspot activity in the second half of 2002 is followed by a quiet time during which the facular area remains at moderately high values. TSI reached a maximum when sunspot activity declined, a few months after chromospheric indices that peaked instead when sunspot emergence was high. Again, the competitive effect of bright and dark regions on the solar disk determines the value of TSI. Consequently, TSI may reach its maximum value at a different time than indices related to specific features such as sunspots and faculae.

\section{RADIATIVE OUTPUT IN SOLAR CYCLE 23: CURRENT OBSERVATIONS AND THEIR IMPROVEMENT}

To study the Sun's radiative output in cycle 23, we extend our analysis (Paper I) through the maximum phase of cycle 23 by using the new TSI measurements and the new photometric indices reflecting effects of solar magnetic regions on the TSI. In the last two years both the irradiance measurements from space and photometric quantities obtained from ground-based observations changed significantly. Photometric calibrations have been reanalyzed and instrumental problems corrected as described below.

\subsection{Total Solar Irradiance}

The latest VIRGO TSI data have been corrected for degradation caused by exposure to UV radiation and exposureindependent changes (Fröhlich 2003a, 2003b) The sensitivity change caused by exposure to radiation in flight is corrected by comparing the radiometer used for daily measurements with an identical radiometer that is exposed infrequently. Exposureindependent changes are more difficult to identify and to correct (Fröhlich 2003a). The VIRGO experiment includes two different types of radiometers, PMO6 and DIARAD, that take simultaneous measurements. Comparison of the time series from the two instruments allows us to correct for such changes as described in Fröhlich (2003a, 2003b). The VIRGO data have also been validated against the ACRIMSat/ACRIMIII measurements available from R. C. Willson (2003, private communication) for the period April 2000 to September 2003. TSI time series from these two space experiments agree well within the errors of the measurements (de Toma et al. 2004). The rms difference between these two TSI records is $0.12 \mathrm{~W}$ $\mathrm{m}^{-2}$, or $88 \mathrm{ppm}$, and the average difference is less than $0.5 \mathrm{~W}$ $\mathrm{m}^{-2}$. Both TSI time series show the three relative maxima in 

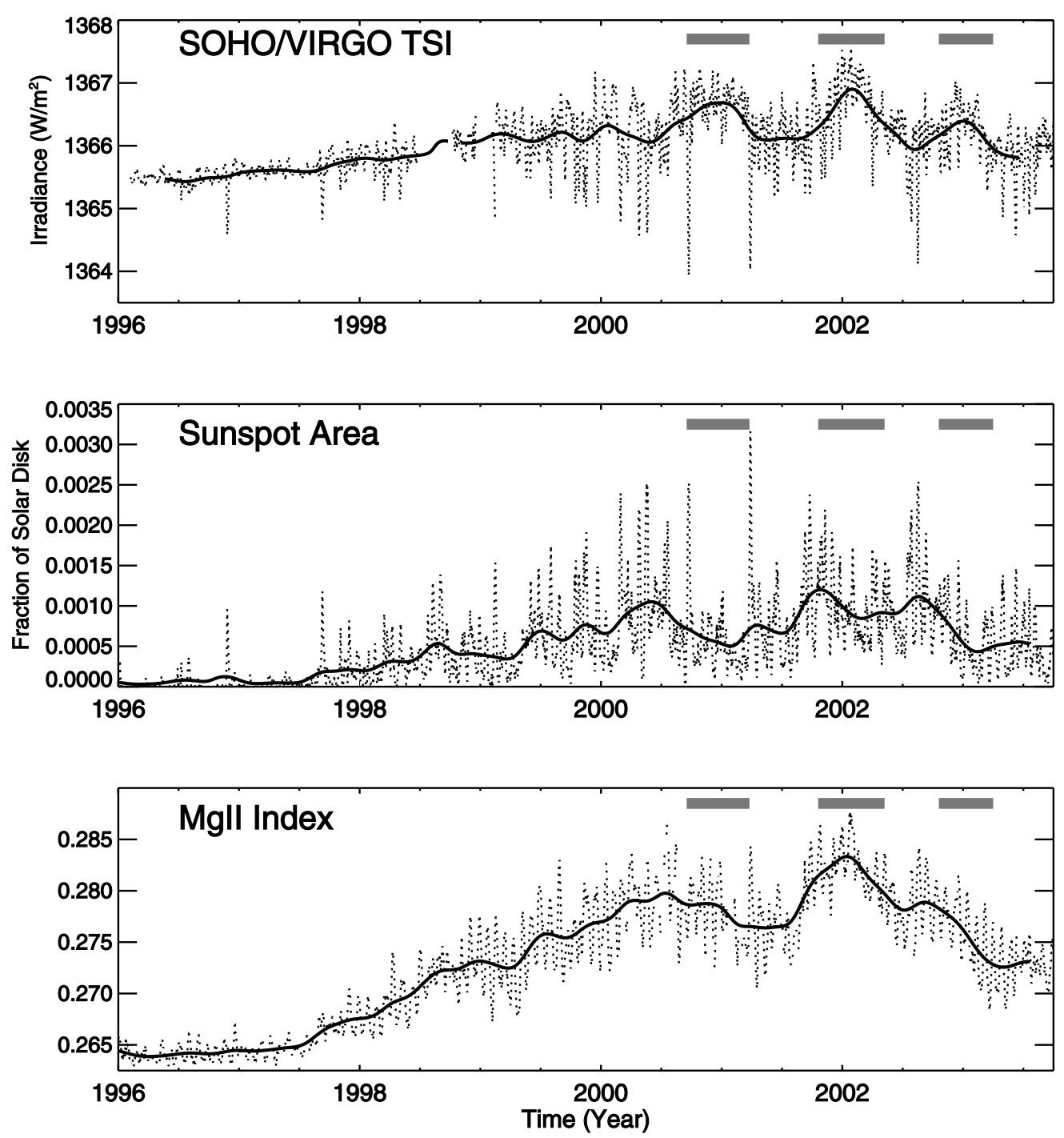

FIG. 2.-Time series of daily values for TSI irradiance as observed by SOHO VIRGO during solar cycle 23 (top), total sunspot area for the day as computed from the SOON network database (middle), and composite $\mathrm{Mg}$ II index from space data (bottom). The sunspot area is given here as the fraction of the solar disk (instead of hemisphere) for easier comparison with the irradiance data. Of particular interest is the period between 2000 October and 2001 March marked by the first gray bar. This period, bracketed by the two largest sunspot groups of cycle 23, shows very low sunspot emergence. The magnetic flux and other indices of solar activity show relatively low values at this time, while TSI stays high.

the period from 2000 October into early 2003 discussed in the previous section.

The new VIRGO TSI time series differs significantly from the one available to us at the time of our first analysis and does not show the rapid increase reported in Paper I and in Frölich \& Finsterle 2001 for the ascending phase of cycle 23. Improvements in the calibration of the VIRGO data are described by Fröhlich $(2000,2002,2003 \mathrm{~b})$ and include correction for exposure-independent changes in the VIRGO radiometers that were not fully taken into account in the earlier calibrations. Variation from minimum to maximum during cycle 23 is now $0.85 \mathrm{~W} \mathrm{~m}^{-2}$, while earlier versions of the VIRGO data showed an increase of about $1.0 \mathrm{~W} \mathrm{~m}^{-2}$. The $0.85 \mathrm{~W} \mathrm{~m}^{-2}$ increase in cycle 23 is comparable to the $\approx 0.80 \mathrm{~W} \mathrm{~m}^{-2}$ increase observed in cycle 22.

\subsection{Indices for Solar Irradiance Modeling}

Empirical models of TSI commonly use measures of "sunspot darkening" and "facular brightening" (Lean \& Fröhlich 1998; Lean 2000, and references therein). Their use in TSI analysis assumes that solar magnetic features such as sunspots, faculae, plages, and chromospheric network are the main sources of solar irradiance variability. Here we use photometric indices developed in our image analysis program at SFO that include indices for bright and dark solar structures, as well as new indices for irradiance variability. The new indices derived at SFO are sums of all pixels in full-disk solar images recorded at two wavelengths at which sunspots and faculae show in photospheric radiation and bright plages and network are clearly seen in the $\mathrm{Ca}$ II $\mathrm{K}$ line. Such full-disk quantities differ from those indices for which magnetic surface features are identified and measured individually because they contain information from all structures visible on the solar disk, including the "quiet Sun." The following paragraphs define in more detail all the variability measures from SFO, as well as describe the Mg II $280 \mathrm{~nm}$ index obtained from UV spectral irradiance measurements from space experiments.

To account for effects of sunspots, faculae, and plages on solar irradiance individually, we use photometric quantities derived from SFO filtergrams (Chapman et al. 1992; Preminger 
et al. 2002): (1) the calcium photometric excess, $E_{K}$, derived from pixels identified as plages and bright network in $\mathrm{Ca}$ II $\mathrm{K}$ line images, (2) the red photometric excess, $E_{r}$, derived from pixels identified as faculae in continuum images recorded at $672.3 \mathrm{~nm}$, and (3) the red photometric deficit, $D_{r}$, due to pixels identified as sunspots in continuum images recorded at $672.3 \mathrm{~nm}$ (Chapman et al. 1996; Preminger et al. 2001). Such measures require the identification of dark and bright features in calibrated full-disk images consistently throughout the solar cycle. The SFO images are first calibrated and then divided by the mean limb-darkening function to produce a contrast image (Walton et al. 1998; Walton \& Preminger 1999). In this way images are put on a relative intensity scale. Then, a decomposition algorithm based on intensity contrast and contiguity (Preminger et al. 2001) is applied to identify solar features (Walton et al. 2003; Preminger et al. 2002). In these SFO photometric quantities, each solar structure is accounted for with its measured area and contrast. In this respect, they differ from other solar indices, such as the photometric sunspot index PSI (Fröhlich et al. 1994; Lean et al. 1998; Lean 2000), that are not derived from decomposition of photometrically calibrated full-disk images but are instead computed using area estimates and a modeled contrast.

Preminger et al. (2002) introduce the photometric sum $\Sigma$, the sum of all pixels in a full-disk calibrated image. Thus, $\Sigma$ measures the relative spectral irradiance change in a specific wavelength band defined by an optical filter. Three new photometric indices, $\Sigma_{r}, \Sigma_{b}$, and $\Sigma_{K}$, are computed from fulldisk images in the red continuum $(672.3 \mathrm{~nm}, 10 \mathrm{~nm}$ bandpass), the blue continuum (472.3 nm, $10 \mathrm{~nm}$ bandpass), and the Ca II K line (393.4 nm, $1 \mathrm{~nm}$ bandpass), respectively. The sums from the two continuum images measure irradiance contributions from structures in the photosphere, while $\Sigma_{K}$ computed from $\mathrm{Ca}$ II $\mathrm{K}$ images contains information on variability due to chromospheric plages and active network. Such integral quantities contain contributions from all features on the solar disk, including those with contrast too low to be identified as individual features by image decomposition algorithms.

We also use the $\mathrm{Mg}$ II $280 \mathrm{~nm}$ core-to-wing ratio that inherently contains information from the entire solar disk. This index is very relevant to irradiance analysis since it is derived from daily UV irradiance measurements at $280 \mathrm{~nm}$ made continuously since 1978 by several space experiments (Viereck et al. 1999 and references therein). The Mg II line cores originate in the chromosphere and show variability due to faculae and plages similar to that seen in the NSO Ca II $\mathrm{K}$ indices. Because these indices are referenced to irradiances measured in the line wings, they are insensitive to instrumental degradation and the presence of sunspots. The Mg II index, which is measured from space, also has the advantage of missing very few data points compared with records from ground-based observations.

In the following analysis, we use linear combinations of these measures of irradiance variability due to photospheric features (sunspots and faculae), together with a measure of UV variability from bright features (plages and network) in our regression analysis of TSI variation in cycles 22 and 23. Using "sunspot-darkening" and "facular-brightening" types of indices to model TSI rests on the assumptions that bright and dark magnetic features are responsible for solar irradiance variability and that a linear combination of these magnetic indices can reproduce TSI variability well. Using indices based on full-disk integrals to model TSI assumes that the variability of the entire solar spectrum can be modeled by a linear combination of indices measured at specific chromospheric and photospheric wavelengths.

\subsubsection{Comparison of Solar Activity Indices}

The composite Mg II index used in this study is an extension of the Mg II composite made by Donnelly et al. (1994) and is constructed from different $\mathrm{Mg}$ II indices produced independently by several space instruments: UARS/SUSIM, UARS/ SOLSTICE, GOME, NOAA9/SBUV2, and NOAA16/SBUV2. The various indices are put on a common scale and then combined in a weighted average according to the method described in de Toma et al. (1997) and White et al. (1998). Our Mg II composite is consistent with but independent of the NOAA/ SEC Mg II composite (the linear correlation coefficient is 0.998). The main differences between the two composites are slightly lower values of our Mg II composite in 1996 during the solar minimum and a lower scaling factor for the NOAA16/SBUV2 data (2001 to the present). Differences before 1996 are negligible. Use of the NOAA/SEC Mg II composite in our empirical fits to TSI has been tested and does not change our results. Note that in the regression analysis presented in $\S 4$ we do not use the Mg II index in its original scale but instead $\left(\mathrm{Mg}\right.$ II $\left.-\mathrm{Mg} \mathrm{II}_{\min }\right) / \mathrm{Mg} \mathrm{II}_{\min }$, where $\mathrm{Mg} \mathrm{II}_{\min }$ is the average value of the $\mathrm{Mg}$ II index at solar minimum, 0.263. With this scaling, the constant term in our fitting equations represents the value of TSI at solar minimum.

A comparison of the $\mathrm{Mg}$ II index with SFO $\mathrm{Ca}$ II $\mathrm{K}$ indices revealed instrumental changes over time in the two SFO telescopes (CFDT1 and CFDT2). During the past year, the SFO team conducted a careful analysis of the SFO instruments and found the SFO CFDT1 calcium filter began to deteriorate in 1996, and its degradation accelerated in 1999. The CFDT2 $1.0 \mathrm{~nm}$ filter also showed significant degradation from 1992 to when it was replaced in 1998. However, the CFDT2 narrow calcium filter has been stable since its installation, and it is used as a reference to characterize the changes in the other filters and to compensate for their effect. In this way, a new and more consistent data set of photometric $\mathrm{Ca}$ II $\mathrm{K}$ indices has been produced at SFO. A more extended discussion of this calibration procedure is in preparation (G. A. Chapman \& S. R. Walton 2004, private communication). Our present analysis therefore includes the latest versions of the SFO photometric $\mathrm{Ca}$ II $\mathrm{K}$ indices determined from analysis of the CFDT1 and CFDT2 images.

The SFO $\Sigma_{K}$ and the NSO Kitt Peak Ca II K index rise more slowly in cycle 23 than in cycle 22 . This slower rate of increase is also observed in other measures, such as the SFO sunspot area, the SOON sunspot area, the $10.7 \mathrm{~cm}$ radio flux, and the NSO Kitt Peak He I $1083 \mathrm{~nm}$ index, as well as in the NSO Kitt Peak photospheric magnetic flux. However, the Mg II $280 \mathrm{~nm}$ index shows only a small reduction in the minimumto-maximum increase in cycle 23 (see Table 1). The scatter plot of the NSO Kitt Peak Ca II $\mathrm{K}$ index versus the Mg II index in Figure 3 illustrates this systematic difference, in spite of the high correlation between the two indices. The squares (cycle 23) and circles (cycle 22) lie along two different linear correlation lines in the sense that the NSO Kitt Peak Ca II K index is systematically lower in cycle 23 relative to cycle 22 for a given value of the $\mathrm{Mg}$ II index. Since we do not know whether the differences between the $\mathrm{Mg}$ II index measured from space and the $\mathrm{Ca}$ II $\mathrm{K}$ indices from ground-based experiments are real or caused by unknown instrumental problems, we use both the $\mathrm{Mg}$ II index and the SFO $\Sigma_{K}$ in our regression analysis. In 

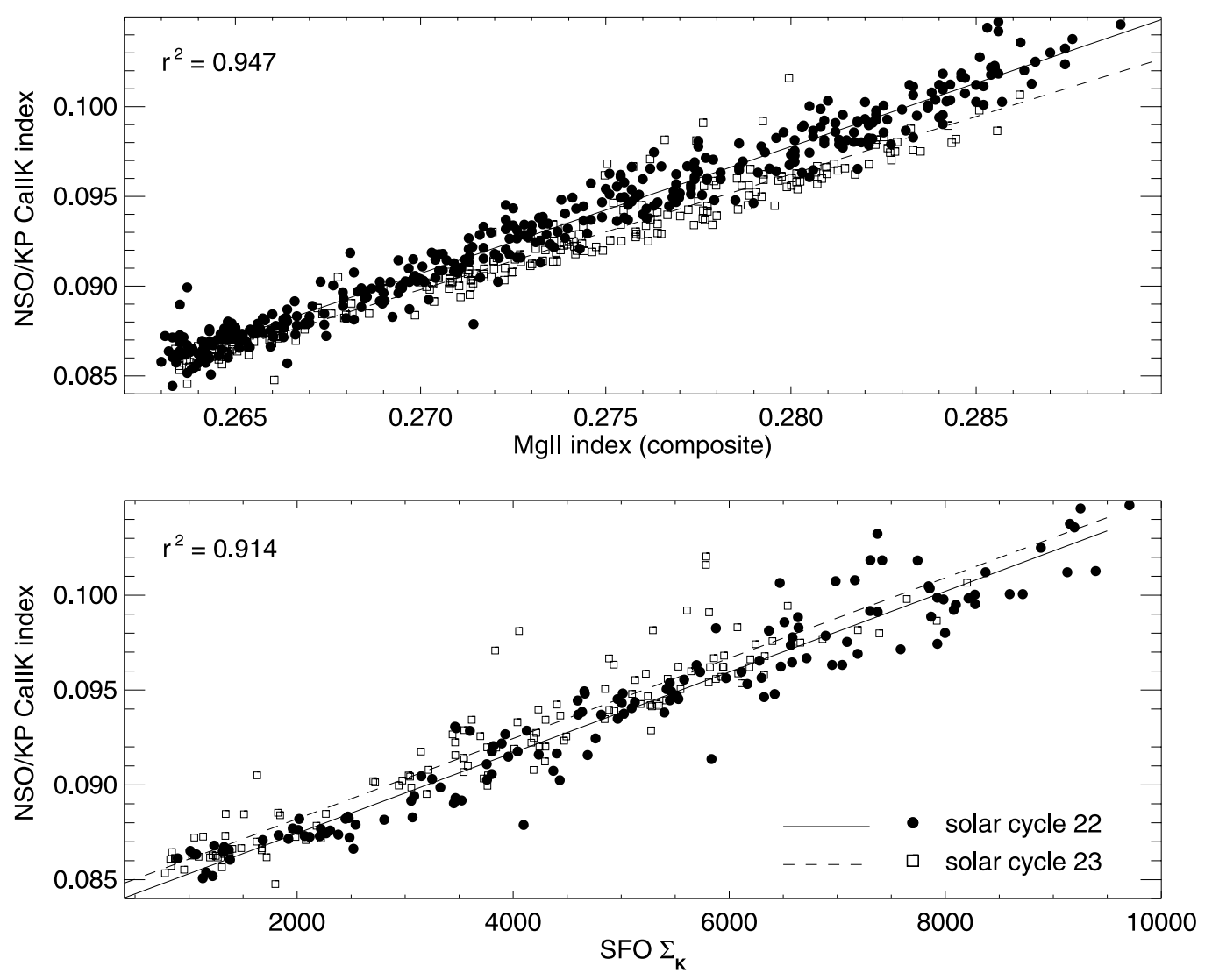

Fig. 3. - Comparison of the Mg II composite and $\Sigma_{K}$ photometric index with the NSO Kitt Peak Ca II K index. Circles are used for cycle 22 and squares for cycle 23. The straight-line fits to the indices for cycle 22 (continuous lines) and cycle 23 (dashed lines) show a different linear relationship between the Mg II index and the NSO Kitt Peak Ca II K index in the two cycles, while the SFO $\Sigma_{K}$ index is more consistent with the NSO Kitt Peak Ca II K index during both cycles.

the next section, we discuss the consequences of these differences in empirical fits to the TSI record.

\section{EMPIRICAL ANALYSIS OF TSI}

Here we show that the improved calibration of TSI observations and indices of radiative variability, together with the development of new full-disk indices, improves the empirical fit to the observed TSI records for both cycles 22 and 23. A complete summary of fitting coefficients, correlations coefficients, and rms is given in Table 2. Selected examples of our fits are shown in Figures 4 and 5.

Figure 4 gives four examples of our regressions to the TSI record for the rising phase of cycles 22 and 23. This extends our analysis in Paper I to the maximum phase of cycle 23 and takes advantage of new data. Each panel contains the fitting equations, correlation coefficients, and the rms residuals between observed and modeled TSI. We now find better agreement between empirical models and TSI observations than in Paper I. Using Mg II and SFO $\Sigma_{r}$ gives the best fit to the TSI records for cycles 22 and 23 (see Fig. 4, top panels), with rms residuals of 156 and $96 \mathrm{ppm}$, respectively. Fitting with the SFO photometric facular excess $E_{K}$ and sunspot deficit $D_{r}$ (see Fig. 4, bottom panels) gives significantly lower correlations and higher rms residuals than in the analysis using $\mathrm{Mg}$ II and SFO $\Sigma_{r}$. We also find that using $\Sigma_{K}$ instead of $\mathrm{Mg}$ II in a third example (see Table 2) is intermediate between the two cases shown in Figure 4. Correlation coefficients for three regression models using $\mathrm{Mg}$ II and $\Sigma_{r}, \Sigma_{K}$ and $\Sigma_{r}$, and $E_{K}$ and $D_{r}$ show recovery of $93 \%, 88 \%$, and $82 \%$, respectively, of TSI variability observed in cycle 23 . This systematic decrease in correlation shows that regression models using the $\mathrm{Mg}$ II index and the SFO photometric full-disk indices $\left(\Sigma_{K}\right.$ and $\left.\Sigma_{r}\right)$ recover more of the TSI variability than those based on identification of sunspots and plages $\left(E_{K}\right.$ and $\left.D_{r}\right)$, confirming the conclusion by Preminger et al. (2002). We find a similar improvement in correlation between cycles 22 and 23. Our regression model using $\mathrm{Mg}$ II and $\Sigma_{r}$ recovers $93 \%$ of TSI variability in cycle 23 compared with $86 \%$ for cycle 22 . This reflects the improvement in both the TSI record and measurements of the indices. Furthermore, the rms residuals are comparable to the accuracy of current TSI data.

We do not find the large difference in the fitting coefficients between cycle 22 and 23 reported in Paper I. When we use the new full-disk index $\Sigma_{r}$, together with $\mathrm{Mg}$ II, similar fitting equations represent TSI variations well in both cycle 22 and 23 (see Fig. 4; Table 2). Specifically, the fitting coefficients for $\Sigma_{r}$ agree within $15 \%$ in both cycles, and the fitting coefficient for the $\mathrm{Mg}$ II index is almost unchanged. A discrepancy remains in the fitting equation coefficients when sunspot deficit and facular excess, or $\Sigma_{r}$ and $\Sigma_{K}$, are used. This is less than previously found in fits with $\Sigma_{K}$ but is still a significant difference. We note the small upward trend in the residuals (see Fig. 5) from 1996 to 2003 of about $0.15 \mathrm{~W} \mathrm{~m}^{-2}$ when $\Sigma_{K}$ is used instead of $\mathrm{Mg}$ II, but there is no significant trend in the fit with $\mathrm{Mg}$ II (less than $0.05 \mathrm{~W} \mathrm{~m}^{-2}$ ). This is because $\Sigma_{K}$ has a slower increase from minimum to maximum during cycle 23 than the $\mathrm{Mg}$ II index, as noted in the previous section. For the ascending phase of cycle 23 larger coefficients are necessary to fit TSI well with the SFO indices. The difference found for the SFO $E_{K}$ and $D_{r}$ can be caused, in part, by decreased feature 
TABLE 2

Summary of Fitting Parameters: TSI $=S_{0}(1+a x+b y+c z)$

\begin{tabular}{|c|c|c|c|c|c|c|c|c|c|c|c|c|}
\hline \multirow[b]{3}{*}{ TSI OBSERVATIONS } & \multicolumn{3}{|c|}{1} & \multirow{3}{*}{$\begin{array}{c}2 \\
\text { Constant } \\
S_{0}\end{array}$} & \multirow{2}{*}{\multicolumn{3}{|c|}{3}} & \multirow{2}{*}{\multicolumn{2}{|c|}{4}} & \multirow{2}{*}{\multicolumn{3}{|c|}{5}} \\
\hline & & & & & & & & & & & & \\
\hline & $r$ & $r^{2}$ & $(\mathrm{ppm})$ & & $\mathrm{Mg}$ II & $\langle\mathrm{Mg}$ пा $\rangle$ & $\operatorname{Mg}$ II $-\langle\mathrm{Mg}$ II $\rangle$ & $\Sigma_{K}$ & $\Sigma_{r}$ & $E_{K}$ & $D_{r}$ & $E_{r}$ \\
\hline \multicolumn{13}{|c|}{ Cycle 23} \\
\hline VIRGO 1996-2003.75 .................... & 0.965 & 0.931 & 96 & 1365.520 & 0.01393 & & & & $1.186 \times 10^{-6}$ & & & .. \\
\hline 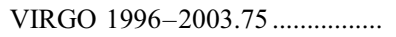 & 0.969 & 0.939 & 91 & 1365.499 & $\ldots$ & 0.0143 & 0.0100 & 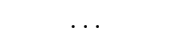 & $1.15710^{-6}$ & $\ldots$ & ... & $\ldots$ \\
\hline 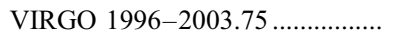 & 0.941 & 0.885 & 123 & 1365.403 & $\ldots$ & $\ldots$ & $\ldots$ & $1.620 \times 10^{-7}$ & $1.061 \times 10^{-6}$ & & & \\
\hline VIRGO 1996-2003.75 ................... & 0.906 & 0.821 & 158 & 1365.289 & $\ldots$ & $\ldots$ & $\ldots$ & $\ldots$ & $\ldots$ & $2.150 \times 10^{-7}$ & $1.457 \times 10^{-6}$ & .. \\
\hline VIRGO 1996-2003.75 …….............. & 0.910 & 0.828 & 155 & 1365.295 & $\cdots$ & $\cdots$ & $\cdots$ & $\cdots$ & $\ldots$ & $2.086 \times 10^{-7}$ & $1.455 \times 10^{-6}$ & $4.400 \times 10^{-6}$ \\
\hline \multicolumn{13}{|c|}{ Cycle $22^{\mathrm{a}}$} \\
\hline Composite $1986-1993.75 \ldots \ldots \ldots \ldots$. & 0.927 & 0.859 & 156 & 1365.553 & 0.01369 & $\ldots$ & $\ldots$ & $\ldots$ & $1.029 \times 10^{-6}$ & $\ldots$ & $\ldots$ & $\ldots$ \\
\hline Composite $1986-1993.75 \ldots \ldots \ldots \ldots$ & 0.931 & 0.867 & 153 & 1365.521 & $\ldots$ & 0.0142 & 0.0104 & & $1.001 \times 10^{-6}$ & & & $\ldots$ \\
\hline Composite $1988-1993.75^{\mathrm{b}} \ldots \ldots \ldots \ldots$ & 0.913 & 0.834 & 178 & 1365.503 & $\ldots$ & $\ldots$ & $\ldots$ & $1.231 \times 10^{-7}$ & $0.860 \times 10^{-6}$ & & & $\ldots$ \\
\hline Composite $1988-1993.75^{\mathrm{b}} \ldots \ldots \ldots \ldots$ & 0.876 & 0.767 & 218 & 1365.415 & $\ldots$ & $\ldots$ & $\ldots$ & $\ldots$ & $\ldots$ & $1.479 \times 10^{-7}$ & $1.152 \times 10^{-6}$ & \\
\hline Composite $1988-1993.75^{\mathrm{b}} \ldots \ldots \ldots \ldots$ & 0.884 & 0.781 & 211 & 1365.426 & $\cdots$ & $\cdots$ & $\ldots$ & $\ldots$ & $\ldots$ & $1.385 \times 10^{-7}$ & $1.137 \times 10^{-6}$ & $4.328 \times 10^{-6}$ \\
\hline \multicolumn{13}{|c|}{ Cycles $22^{\mathrm{a}}$ and 23} \\
\hline Composite $1986-2003.75 \ldots \ldots \ldots \ldots$ & 0.938 & 0.880 & 132 & 1365.503 & 0.01372 & $\ldots$ & .. & $\cdots$ & $1.076 \times 10^{-6}$ & ... & $\ldots$ & $\ldots$ \\
\hline Composite $1986-2003.75 \ldots \ldots \ldots \ldots$ & 0.942 & 0.887 & 129 & 1365.483 & $\ldots$ & 0.0141 & 0.0101 & & $1.046 \times 10^{-6}$ & $\ldots$ & & $\ldots$ \\
\hline Composite $1988-2003.75^{\mathrm{b}} \ldots \ldots \ldots \ldots$ & 0.923 & 0.852 & 152 & 1365.382 & $\ldots$ & $\ldots$ & $\ldots$ & $1.437 \times 10^{-7}$ & $0.931 \times 10^{-6}$ & $\ldots$ & t & $\ldots$ \\
\hline Composite $1988-2003.75^{\mathrm{b}} \ldots \ldots \ldots \ldots$ & 0.869 & 0.755 & 200 & 1365.321 & $\ldots$ & $\ldots$ & $\ldots$ & $\ldots$ & $\ldots$ & $1.679 \times 10^{-7}$ & $1.201 \times 10^{-6}$ & $\ldots$ \\
\hline Composite $1988-2003.75^{\mathrm{b}} \ldots \ldots \ldots .$. & 0.873 & 0.762 & 197 & 1365.335 & $\ldots$ & $\ldots$ & $\ldots$ & $\ldots$ & $\ldots$ & $1.595 \times 10^{-7}$ & $1.196 \times 10^{-6}$ & $4.003 \times 10^{-6}$ \\
\hline
\end{tabular}

NoтEs.-The table lists the coefficients for five different trial functions applied to three TSI time series: cycle 22 and 23 alone and the entire TSI composite covering both cycles 22 and 23 . The first column, labeled

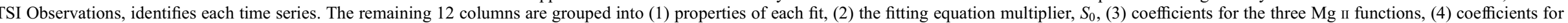

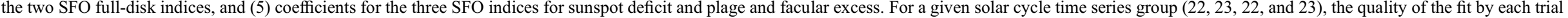

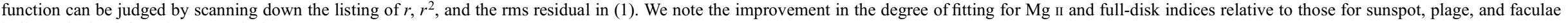
indices obtained from image analysis.

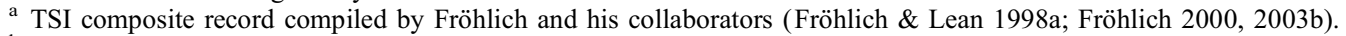

b The SFO $\Sigma_{K}$ and $E_{K}$ photometric indices are available only since 1988. 

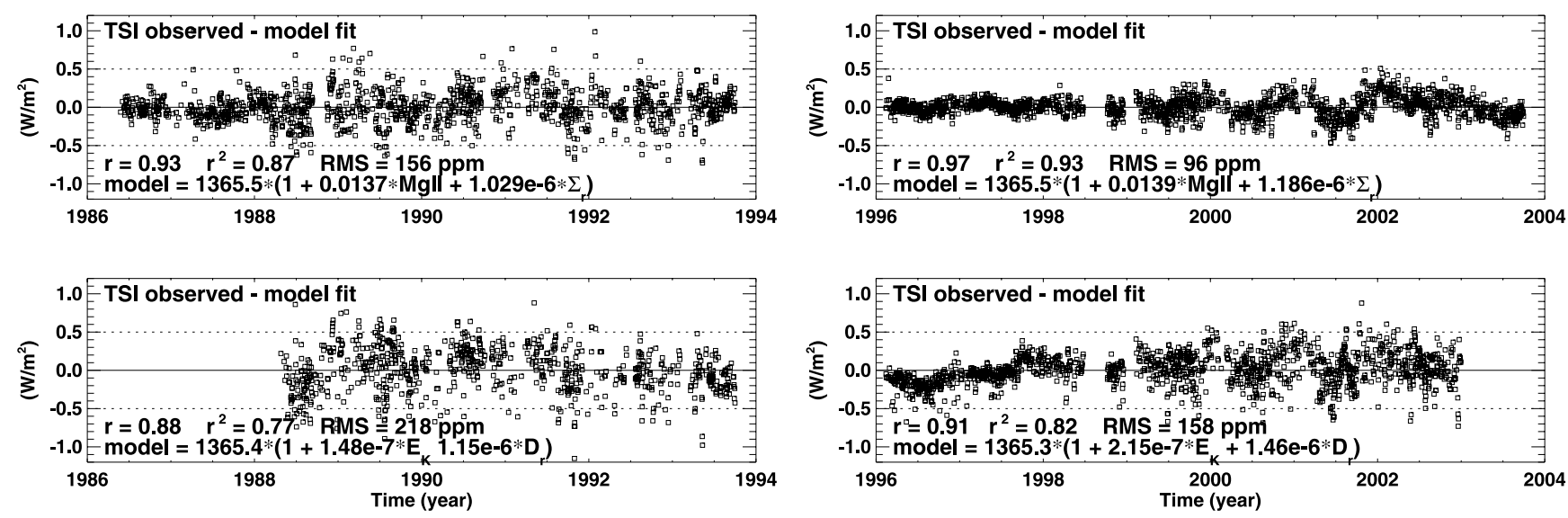

Fig. 4.-Examples of empirical models using full-disk Mg II and $\Sigma_{r}$ indices (top panels) and the SFO facular excess $E_{K}$ and sunspot deficit $D_{r}$ (bottom panels) for solar cycle 22 (left) and 23 (right). We give the linear correlation coefficient, the rms deviation of the residuals between the fit and TSI, and the fitting function in each panel.

contrast due to the instrumental changes in the SFO Ca II K filters mentioned above or by a larger percentage of fainter and then unidentified faculae or plages and sunspots on the solar disk during cycle 23. However, differences in the coefficients when $\Sigma_{K}$ and $\Sigma_{r}$ are used cannot be explained by a change in feature contrast, since those sums include all features regardless of their contrast.

Figure 5 illustrates our results when we fitted the entire TSI composite as a single time series from the solar minimum between cycles 21 and 22 in 1986 to the maximum of cycle 23 . We chose the TSI composite record compiled by Fröhlich and his collaborators (Fröhlich \& Lean 1998a; Fröhlich 2000, 2002, 2003a). The TSI composite is shown in Figure 5 (top), together with a weighted average (thick line) over about six solar rotations using a Gaussian function with an 81 day FWHM. We show the residuals and their weighted average after fitting TSI with $\mathrm{Mg}$ II and SFO $\Sigma_{r}$ (middle panels) and with the SFO full-disk indices $\Sigma_{r}$ and $\Sigma_{K}$ (bottom). We find that all models fit the 27 year TSI record well and to a degree intermediate to fitting the two cycles separately. This is in spite of the difference in solar activity between the two cycles.

In addition to the TSI fits presented above, we made regressions using combinations of three indices. First, following the work of Fröhlich \& Lean (1998b) and Lean (2000), we separated the $\mathrm{Mg}$ II index time series into two components: a low-frequency component, $\langle\mathrm{Mg}$ II $\rangle$, and the difference, $\mathrm{Mg}$ II $-\langle\mathrm{Mg}$ II $\rangle$. The $\langle\mathrm{Mg}$ II $\rangle$ is an average of $\mathrm{Mg}$ II daily values weighted with a Gaussian function with FWHM of 81 days, which effectively is a low-pass filter. The time series $\mathrm{Mg}$ II$\langle\mathrm{Mg}$ II $\rangle$ includes the higher frequency components, such as the rotational period of about 27 days. Since patches of bright network have a longer lifetime than active region plages, the $\langle\mathrm{Mg}$ II $\rangle$ is associated with the chromospheric network, while the difference $\mathrm{Mg}$ II $-\langle\mathrm{Mg}$ II $\rangle$ describes the short-term variability in plages. However, our separation of the $\mathrm{Mg}$ II time series relies on known differences in lifetimes and does not use feature identification. Also, we do not simulate the center-tolimb brightening in faculae, which Fröhlich (2002) takes into account by convolving the TSI+PSI with a double-peaked filter function and $\mathrm{Mg}$ II $-\langle\mathrm{Mg}$ II $\rangle$ with a triangular function. We find that the smoothed $\langle\mathrm{Mg}$ II $\rangle$ term has almost the same coefficient as that for the unsmoothed $\mathrm{Mg}$ II time series. The coefficient of the high-frequency component of the $\mathrm{Mg}$ II time series, $\mathrm{Mg}$ II $-\langle\mathrm{Mg}$ II $\rangle$, is $40 \%$ smaller than that for the lowfrequency component, $\langle\mathrm{Mg} \mathrm{II}\rangle$. This difference in weights between low- and high-frequency $\mathrm{Mg}$ II index contributions to TSI variability shows that more persistent structural features, such as patches of active network and plages, determine the fits over a solar cycle. The high-frequency component contains information on the evolution of bright structures as they move across the solar disk, and it slightly improves the day-to-day representation of TSI variability without changing the weight of the longer lived structures. In a second trial, we added a third term, $E_{r}$, to the fit with $E_{K}$ and $D_{r} . E_{r}$ is based on identification of bright regions in the SFO red continuum images, so it provides a proxy for the photospheric faculae. We expected that decomposing full-disk indices into terms associated with known features on the Sun would give a better representation of TSI. The results of our fits are given in Table 2. In both cases, we saw only a marginal improvement in the goodness of the fit when three indices are used instead of two and the longterm trend in the residuals did not change. We thus conclude that decomposition of existing full-disk indices does not improve the TSI representation significantly. Photometric quantities based on full-disk sums are preferred in empirical models of TSI since they are easily computed and seem to be less sensitive to instrumental problems or seeing effects than quantities requiring feature identification.

The larger scatter in residuals in our TSI fits for cycle 22 relative to cycle 23 shows the improvement in observational accuracy of the TSI record starting in 1996. The rms values for the residuals are always smaller for the same fitting equations for cycle 23 than for cycle 22, as reported in Table 2. Nevertheless, there is a systematic wavelike pattern with an approximate 1 year period in the residuals for all our cycle 23 fits. This pattern begins in early 2000 and extends to the end of the record in 2003. We estimated a mean peak-to-peak amplitude of about $0.4 \mathrm{~W} \mathrm{~m}^{-2}$, or $300 \mathrm{ppm}$, from data in the second panel of Figure 5. This quasi-annual pattern is not peculiar to the VIRGO TSI data. Our fit to the ACRIMIII TSI data during the maximum phase of cycle 23 shows the same pattern in the residuals regardless of which combination of indices we use. This oscillatory pattern mimics the shape of the smoothed TSI record: the three relative maxima in 2000 , 2001, and 2003 in the residuals correspond to relative maxima in TSI values (see Fig. 5). 

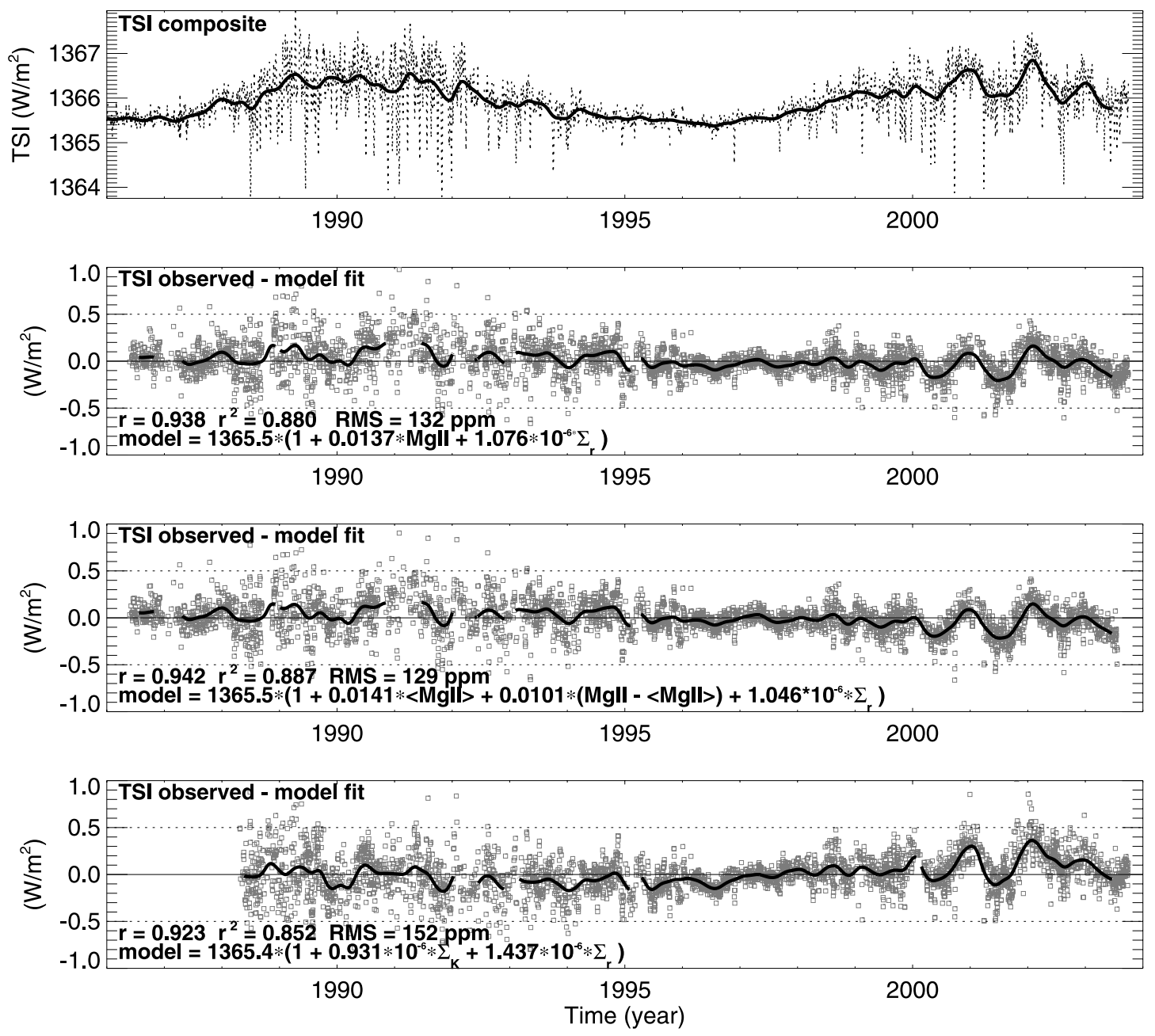

FIG. 5.-TSI composite record from 1986 to 2003 (Fröhlich 2002) constructed by merging observations from different satellites (top), and difference between TSI and its estimates based on empirical models using Mg II and the SFO $\Sigma_{r}$ (middle panels) and SFO $\Sigma_{K}$ and SFO $\Sigma_{r}$ (bottom). The smooth curves are computed using a Gaussian smoothing function with an FWHM of 81 days.

We also note that these three maxima occur early in each year close to the time of perihelion in the Earth's orbit around the Sun. Seasonal variations in the distance from the Sun are accurately accounted for in TSI observations (W. Thompson \& G. Kopp 2003, private communication), so we discount them as the cause of this 1 year pattern. Temperature variations in the spacecraft environment caused by variations in the distance from the Sun can affect the radiometer's response. These temperature effects are more difficult to correct and may contribute to the quasi-annual period in the residuals. Since this periodicity is not present in the residuals in fits to VIRGO TSI data before 2000 and after mid 2003, temperature changes are not a likely cause of the annual TSI variation observed in the maximum phase of cycle 23. Mordvinov \& Willson (2003) suggest that the periodicity originates in the annual variation in the apparent position of the solar poles and differences in activity between the northern and southern hemispheres. Since the indices and photometric parameters used in our fitting process automatically include such asymmetries, the presence of the oscillation in our residuals strongly suggests that it originates in the TSI itself. Continuation of the quasi-annual TSI variation in the remainder of cycle 23 as the level of sunspot activity changes will be an important clue to its origin.

For the pattern in the occurrence of active regions to be the source of this effect, sunspots and their associated faculae must combine to produce a recurrent pattern at approximate 1 year intervals starting late in 1999 and continuing through solar maximum into early 2003. Active region complexes last for several months, so an approximately annual periodicity is possible in solar records. The full radiative effect of TSI that results from sunspot activity is not captured fully by our use of indices and parameters derived directly from daily irradiance measurements (Mg II) and photometric fulldisk images $\left(\Sigma_{K}\right.$ and $\left.\Sigma_{r}\right)$; the disagreement is obvious during this period because of the character of the variations in activity and the much lower noise in the TSI measurements themselves when compared with cycle 22 . The peak-topeak amplitudes in the smoothed TSI data for 2001, 2002, and 2003 are about $0.62,0.84$, and $0.45 \mathrm{~W} \mathrm{~m}^{-2}$, respectively. Amplitudes of the corresponding smoothed residuals are about $0.26,0.37$, and $0.09 \mathrm{~W} \mathrm{~m}^{-2}$, which are $42 \%, 44 \%$, and $19 \%$ of these three TSI excursions, respectively. Thus, our regression analysis recovers from $56 \%$ to $81 \%$ of the peak-topeak amplitude of quasi-annual TSI variation between 2001 and 2003.

Figure 6 shows how negative irradiance variations in the $\Sigma_{r}$ parameter (second panel) can interact with the positive contributions in the $\Sigma_{k}$ or Mg II $280 \mathrm{~nm}$ index (two bottom panels) to produce the quasi-annual TSI variation (top) seen between 2000 and 2003 in cycle 23. The larger negative contributions 
Total Solar Irradiance (composite)
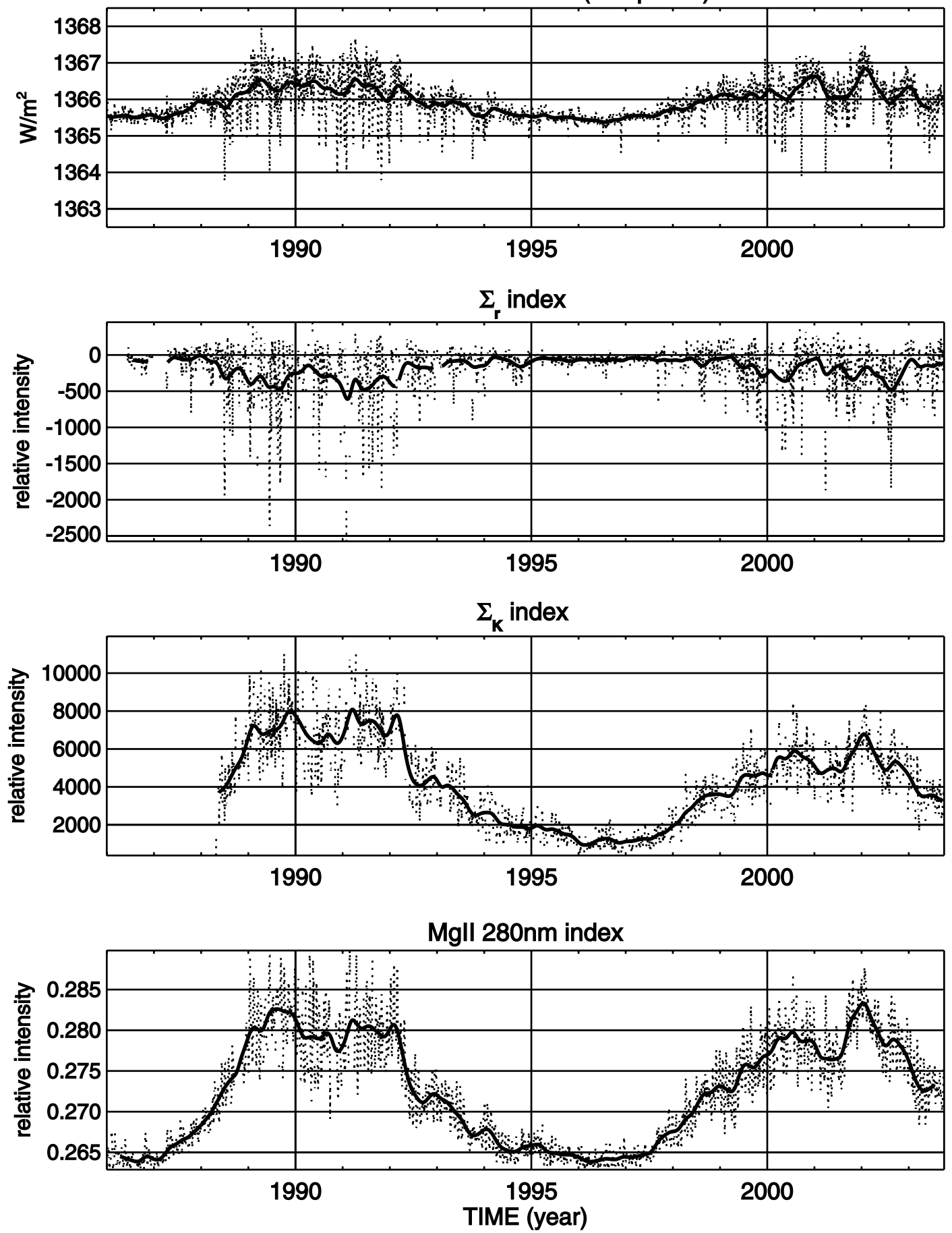

FIG. 6.-TSI composite record from 1986 to 2003 (top; Fröhlich 2002), and indices for irradiance (bottom): SFO $\Sigma_{r}$, SFO $\Sigma_{K}$, and Mg II. We plot daily values, as well as averages obtained with a Gaussian filter of 81 days FWHM. The relative maxima in TSI correspond to relative maxima in the Ca II and Mg II indices and/or to local maxima in the $\Sigma_{r}$. Large negative values of $\Sigma_{r}$ indicate high sunspot activity.

in $\Sigma_{r}$ tend to bracket the peaks in $\Sigma_{K}$ and $\mathrm{Mg}$ II. This interference between irradiance contributions from dark and bright features results in the quasi-annual TSI variation. This is a chance variation in the emergence of active regions and their evolution in cycle 23 , when fewer such regions occurred. This simplicity in the distribution of active regions on the solar disk helps to understand the quasi-annual TSI variation during the maximum phase of cycle 23. This balance or interference in contributions of bright and dark structures to the TSI changes with time as all components of an active region evolve and vary in appearance as they move across the solar disk.

\section{CONCLUSIONS}

Cycle 23 is weaker than cycle 22, not only in magnetic flux but in most activity indices. Sunspot and facular areas are significantly lower for cycle 23 , by about $37 \%$ and $46 \%$, respectively. TSI is relatively less affected by the low sunspot activity because its variation comes from a balance of dark (sunspots) and bright (faculae, plages, and network) 
components seen in active regions. The lower sunspot area and the excess of small and relatively weak spots in cycle 23 relative to cycle 22 may explain why TSI values remain high during cycle 23 , while most solar activity indices are lower.

Cycle 23 is magnetically simpler than its immediate predecessors, 21 and 22; therefore, the current solar cycle can be considered anomalous only in the sense of decadal timescales and not over centennial timescales since similar weak cycles have been observed. Although cycles 22 and 23 violate the even-odd rule, cycle 23 should not be considered anomalous since historic records based on aurora data suggest that violations of the even-odd rule may have occurred in the past. The even-odd rule is purely empirical, and present dynamo models imply a more complex relationship between cycles.

The period of low sunspot activity between 2000 October and 2001 March does not correspond to a decrease in TSI. The greatly reduced sunspot activity, in combination with moderately high facular activity, seems to be responsible for the relatively high TSI values. This indicates that the relative change in TSI cannot be reproduced well using sunspot indices alone. Since the sunspot number is our only measure of solar activity before 1900, accurate reconstruction of TSI in the past is limited by lack of direct observation of faculae, plages, and bright network.

Well-calibrated photometric quantities derived from full-disk observations, such as the Mg II index and the $\Sigma_{r}$, provide good fits to the VIRGO TSI observations. The composite TSI record can be represented well by a linear regression model with only two components related to magnetic regions. Typical correlation coefficients are on the order of 0.93 or better; thus, these models recover over $86 \%$ of TSI variability. Decomposition of the full-disk indices into two terms representing different structure classes does not seem to improve the quality of the fit to TSI significantly. Nevertheless, image decompositions are useful to understand how different types of solar surface features associated with stronger magnetic fields contribute to observed TSI variability. Higher resolution and higher accuracy instruments, such as PSPT and CFDT3 at SFO, will likely provide higher quality indices for the different structure classes than the older generation of photometric telescopes.

Fitting TSI data in the rising phases of both cycles 22 and 23 with the Mg II index and the new SFO photometric indices used in this paper gives better fits and similar fitting coefficients for both cycles. This answers the question raised in Paper I: We find no need for a third, nonmagnetic, source of TSI variability beyond those used here.

We note a steady improvement in the empirical representations of TSI from cycle 22 to cycle 23 (lower rms values and higher correlation coefficient), corresponding to improvement in the quality of the TSI record, as well as to the development of better solar activity indices. In spite of this improvement, interesting differences between TSI data and our regression models show in the residuals during the rise to the cycle 23 maximum. As noted earlier, during the period 1996-2003 residuals show a small upward trend of $0.15 \mathrm{~W}$ $\mathrm{m}^{-2}$ when $\Sigma_{K}$ is used instead of $\mathrm{Mg}$ II because $\Sigma_{K}$ increases more slowly than the $\mathrm{Mg}$ II index. This a common difference between the $\mathrm{Mg}$ II index from space and the $\mathrm{Ca}$ II indices available to us from the ground. Whether this difference with a solar cycle timescale reflects a physical difference between the two types of observation remains for future research.

The quasi-annual fluctuation seen in TSI observations between 2000 and 2003 is likely a physical property of the TSI due to interference between the negative sunspot contributions and the positive contributions from plages and faculae in cycle 23 and is not an instrumental artifact. It is clearly visible in cycle 23 because of the chance occurrence of fewer active regions than in cycle 22 . The wave-shaped pattern with approximate 1 year period is clearly seen in the residuals during the maximum phase of cycle 23 in all our fits and is a remnant of this quasi-annual TSI variation. We recover between $55 \%$ and $80 \%$ of the peak-to-peak TSI amplitude by using our choice of two parameters to represent variation in radiation from photospheric and chromospheric structures. This suggests that we reached an information limit existing in both our choice of two fitting parameters and the TSI observations themselves. It is not unreasonable to find that the variation in the integral of the solar irradiance spectrum (the TSI) cannot be recovered to better than 100-200 ppm by using measurements in only two wavelength bands, such as the SFO continuum band at $672.3 \mathrm{~nm}$ and the $280 \mathrm{~nm}$ band containing the Mg II doublet.

This research was partially supported by NASA grants S-13782-G and NAG5-12905 and NSF grant ATM-9912132. The sunspot number and the $10.7 \mathrm{~cm}$ radio flux were made available by the NOAA National Geophysical Data Center. The NSO Kitt Peak data used here are produced cooperatively by NSF NOAO, NASA GSFC, and NOAA SEL. The VIRGO TSI data are available from the $S O H O$ VIRGO web site and the ACRIMIII TSI data from the ACRIM web site. We thank Claus Fröhlich and Dick Willson for the information on the TSI data calibration. We thank Bill Thompson (SOHO operations) and Greg Kopp (CU LASP) for the information provided about the accuracy of the satellite-Sun distance computations. We also thank Tom Bogdan for his suggestions, which improved our manuscript. The National Center for Atmospheric Research is sponsored by the National Science Foundation.

\section{REFERENCES}

Chapman, G. A., Cookson, A. M., \& Dobias, J. J. 1996, J. Geophys. Res., 101, 13541

Chapman, G. A., et al. 1992, J. Geophys. Res., 97, 8211

Charbonneau, P., \& Dikpati, M. 2000, ApJ, 543, 1027

de Toma, G., White, O. R., Chapman, G. A., \& Walton, S. R. 2004, Adv. Space Res., in press

de Toma, G., White, O. R., Knapp, B. G., Rottman, G. J., \& Woods, T. N. 1997, J. Geophys. Res., 102, 2597

de Toma, G., et al. 2001, ApJ, 549, L131 (Paper I)

Donnelly, R. F., White, O. R., \& Livingston, W. C. 1994, Sol. Phys., 152, 69

Foukal, P., \& Lean, J. L. 1988, ApJ, 328, 347
Fröhlich, C. 2000, Space Sci. Rev., 94, 15

2002, Adv. Space Res., 29(10), 1409

2003a, in Proc. ISCS Symp., Solar Variability as an Input to the

Earth's Environment (ESA SP-535; Noordwijk: ESA) 2003b, Metrologia, 40, S65

Fröhlich, C., \& Finsterle, W. 2001, in IAU Symp. 203, Recent Insight into the Physics of the Sun and Heliosphere: Highlights from $\mathrm{SOHO}$ and Other Space Missions, ed. P. Brekke, B. Fleck, \& J. Gurman (San Francisco: ASP), 105 Fröhlich, C., \& Lean, J. L. 1998a, in IAU Symp. 185, New Eyes to See the Sun and Stars, ed. D. W. Kurtz \& J. Leibacher (Dordrecht: Kluwer), 89 . 1998b, Geophys. Res. Lett., 25, 4377 
Fröhlich, C., \& Pap, J. M. 1989, A\&A, 220, 272

Fröhlich, C., Pap, J. M., \& Hudson, H. S. 1994, Sol. Phys., 152, 111

Gnevyshev, M. N., \& Ohl, A. I. 1948, AZh, 25, 18

Hathaway, D. H., Wilson R. M., \& Reichmann E. J. 1999, J. Geophys. Res., 104,22375

Joselyn, J., et al. 1997, EOS Trans. AGU, 78, 205, 211

Komitov, B., \& Bonev, B. 2001, ApJ, 554, L119

Lean, J. L. 2000, Space Sci. Rev., 94, 39

Lean, J. L., Cook, J., Marquette, W., \& Johannesson, A. 1998, ApJ, 492, 390

Lean, J. L., \& Foukal, P. 1988, Science, 240, 906

Lean, J. L., \& Fröhlich, C. 1998, in ASP Conf. Ser. 140, Synoptic Solar Physics, ed. K. S. Balasubramaniam, J. W. Harvey, \& D. M. Rabin (San Francisco: ASP), 281

Livingston, W. C. 2002, Sol. Phys., 207, 41
Mordvinov, A. V., \& Willson, R. C. 2003, Sol. Phys., 215, 5

Preminger, D. G., Walton, S. R., \& Chapman, G. A. 2001, Sol. Phys., 202, 53 2002, J. Geophys. Res., 107, 1354

Schove, D. J. 1955, J. Geophys. Res., 60, 127

Viereck, R. A., Puga, L. C., \& Lawrence, C. 1999, J. Geophys. Res., 104, 9995

Walton, S. R., Chapman, G. A., Cookson A. M., Dobias J. J., \& Preminger, D. G. 1998, Sol. Phys., 179, 31

Walton, S. R., \& Preminger, D. G. 1999, ApJ, 514, 959

Walton, S. R., Preminger, D. G., \& Chapman, G. A. 2003, Sol. Phys., 213, 301

White, O. R., de Toma, G., Rottman, G. J., Woods, T. N., \& Knapp, B. G. 1998, Sol. Phys., 177, 89

Willson, R. C., Gulkis, S., Janssen, M., Hudson, H. S., \& Chapman, G. A. 1981, Science, 211, 700

Willson, R. C., \& Hudson, H. S. 1991, Nature, 351, 42 